\title{
GMR
}

\section{Circulating miR-125b as a biomarker of Ewing's sarcoma in Chinese children}

\author{
C.L. Nie ${ }^{1 *}$, W.H. Ren ${ }^{1 *}$, Y. $\mathrm{Ma}^{2}$, J.S. $\mathrm{Xi}^{1}$ and B. Han ${ }^{1}$ \\ ${ }^{1}$ Department of Pediatrics, \\ Shandong Provincial Hospital Affiliated to Shandong University, Jinan, China \\ ${ }^{2}$ Department of Pediatrics, Shandong Pediatric Traffic Hospital, Jinan, China \\ ${ }^{*}$ These authors contributed equally to this study. \\ Corresponding author: B. Han \\ E-mail: bhanjnpe@163.com \\ Genet. Mol. Res. 14 (4): 19049-19056 (2015) \\ Received August 15, 2015 \\ Accepted October 7, 2015 \\ Published December 29, 2015 \\ DOI http://dx.doi.org/10.4238/2015.December.29.12
}

ABSTRACT. Previous studies indicated that microRNA-125b (miR-125b) has an important role in the progression of Ewing's sarcoma (ES). The purpose of the current study was to examine expression changes of miR$125 \mathrm{~b}$ in the serum of ES patients and evaluate if the expression level of miR-125b could serve as a new biomarker for ES. This study was performed on patients who underwent surgical resection at our hospital between 2005 and 2013 after an initial diagnosis of ES. We measured serum miR-125b levels in 63 patients with ES and 126 healthy control patients using a real-time quantitative reverse transcriptase-PCR (qRTPCR) method. Expression levels of serum miR-125b were distinctly decreased in ES patients when compared with healthy controls ( $P$ < 0.001). ES cases that had a poor response to chemotherapy presented a significant down-regulation of miR-125b $(P=0.001)$. The ROC curve showed that the serum miR-125b could serve as a valuable biomarker for differentiating ES patients from healthy controls with an AUC of $0.879(95 \% \mathrm{Cl}=0.817-0.924 ; \mathrm{P}<0.001)$. At a cut-off value of 2.203 for miR-125b, the sensitivity was $72.8 \%$ and the specificity was $87.2 \%$ in 
discriminating ES from the controls. Our results indicate that serum miR$125 b$ may serve as a useful noninvasive biomarker for ES.

Key words: Ewing's sarcoma; miR-125; Biomarker; Serum; Diagnosis

\section{INTRODUCTION}

Ewing's sarcoma (ES) is the second most frequent bone and soft tissue tumor, which is characterized by small, blue round cells (Schleiermacher et al., 2003; Iwamoto, 2007; Esiashvili et al., 2008). It occurs mainly in children and adolescents, with an overall incidence of about 1.3 cases per million people (Dorfman and Czerniak, 1995; Erisir et al., 2008). Over the past decades, there has been a great effort to elucidate the underlying mechanisms of ES development, and to identify novel diagnostic biomarkers and therapeutic targets for ES patients (Borinstein et al., 2013). However, the underlying molecular and cellular mechanisms for ES carcinogenesis remain largely unclear. It is reported that approximately $20 \%$ of individuals with ES have a poor prognosis, with an estimated free survival of less than $20 \%$ (Stahl et al., 2011). Those survivors of sarcomas, with distant metastasis, have lower health quality of life scores and require high cure costs (Barr and Wunder, 2009). Moreover, about $25 \%$ of patients suffering from ES have detectable metastasis at diagnosis. Therefore, the development of novel and more effective diagnostic biomarkers for this malignant disease is imperative.

MicroRNAs (miRs) are a large family of non-coding single-stranded RNAs of 18-25 nucleotides (Bartel, 2004). As a newly discovered class of cellular bioactive molecules, miRs play important roles in the regulation of gene expression both in normal and disease physiologies (Ghildiyal and Zamore, 2009). Currently, it is expected that there are more than 1500 miRs that might influence the expression of over $60 \%$ of human genes (Friedman et al., 2009; Kozomara and Griffiths-Jones, 2011). In recent years, many studies identified aberrantly expressed miRs in most human tumors and demonstrated their important roles in several tumors' pathogenesis and metastasis. In particular, a number of recent studies have detected the biology of miRs in the initiation and progression of ES. A specific miR may have a distinct role in different types of cancer, which could potentially contribute to an important advance in the diagnosis, prognosis, and prediction of therapeutic responses of human cancer (Yu et al., 2007; Fabbri, 2010; Ferracin et al., 2010). Therefore, the application of miR signatures can have a great potential in the identification of novel diagnostic molecular biomarkers for cancer.

miR-125b is a member of the most consistently dysregulated miRs. At present, the role of miR-125b in the progression of ES has been widely studied. De Vito et al. (2011), in their study with 11 normal bone tissues and 23 primary ES tumors under microarray analysis, have demonstrated that the expression of miR-125b decreased in ES tissues when compared to normal bone tissues. lida et al. (2013) reported that the overexpression of miR-125b in parental ES cells leads to enhanced drug resistance via inhibition of the expression of apoptotic mediators, such as p53 and Bak. Moreover, McKinsey et al. (2011) indicated that the ectopic expression of miR-125b results in the negative regulation of the insulin-like growth factor (IGF) signaling pathway and suppression of cell proliferation for A673 cells. All these data indicate the potential effect of miR-125b for the diagnosis and therapy of ES.

However, these findings above are not enough to open a new era of ES management. Therefore, it is urgent that further understanding of aberrant miR expression and exploration of 
novel miR biomarkers for ES can be achieved. The purpose of the current study was to examine expression changes of miR-125b in the serum of ES patients and evaluate whether or not the level of miR-125b could serve as a new biomarker.

\section{MATERIAL AND METHODS}

\section{Patients and sampling}

This study was approved by the Review Board of Shandong Provincial Hospital Affiliated to Shandong University Ethics Committee. Each participant signed a written informed consent. Sixty-three patients, who were diagnosed with ES and received surgery at our hospital between January 2005 and October 2013, were recruited for this study. The mean age of the patients was $14 \pm 4.8$ years and none of them underwent preoperative treatment. Primary tumors location sites included the pelvis $(N=17)$, femur $(N=13)$, tibia $(N=10)$, fibula $(N=9)$, humerus $(N=8)$, spine $(N=1)$, rib $(N=3)$, and foot $(N=2)$. There were nine patients with metastatic disease at first diagnosis. Thirty-four patients responded to chemotherapy. After surgery, all tumor specimens were independently reviewed by two experienced pathologists. Tumor size was determined as the maximum tumor diameter based on radiographic images, including computed tomography (CT) scan and magnetic resonance imaging (MRI). One hundred and twenty six healthy individuals, who undertook routine health examinations, were included in the matched-pair control group based on age and gender with a ratio of 1:2. The characteristics of all subjects, including age, gender, tumor size, tumor site, metastasis, and response to chemotherapy, were collected from reviewing medical records or questionnaires.

Peripheral venous blood samples $(5-10 \mathrm{~mL})$ were obtained before all subjects received surgical resection or other therapeutic treatments. Then, these blood samples were centrifuged at $2000 \mathrm{~g}$ for $10 \mathrm{~min}$, and stored at $-80^{\circ} \mathrm{C}$ until use.

\section{RNA extraction, reverse transcription and qPCR}

Blood samples for miR detection were drawn into EDTA-K2 tubes. RNA extraction was performed using the RNeasy Plus Micro Kit (Qiagen, Netherlands), following the manufacturer instructions. The collected RNA yields were around $250 \mathrm{ng}$ per $400 \mathrm{ml}$ serum. Isolated RNA samples were reverse-transcribed to cDNA using the First Strand cDNA Synthesis Kit (Roche Applied Science, Mannheim, Germany). Concentration and purification of miR were conducted with NanoDrop ND-1000 (Nanodrop, Wilmington, DE, USA).

qRT-PCR analysis of miR-125b expression profiles was performed using TaqMan miRNA assays (Applied Biosystems), following the manufacturer instructions. The primer sequences for miR-125b were: GCUCCCUGAGACCCUAAC (forward) and CAGTGCAGGGTCCGAGGT (reverse); and the PCR primers for U6 were: CTCGCTTCGGCAGCACATATACT (forward) and ACGCTTCACGAATTTGCGTGTC (reverse). The expression level of miR-125b was measured using the $\triangle \Delta^{\mathrm{CT}}$ method. SnRNA $U 6$ was used as the reference miR. Reactions were drawn onto a 96 -well plate and were run in duplicate at $50^{\circ} \mathrm{C}$ for $20 \mathrm{~s}, 95^{\circ} \mathrm{C}$ for $10 \mathrm{~min}$, followed by 40 cycles of $95^{\circ} \mathrm{C}$ for $15 \mathrm{~s}$, and $60^{\circ} \mathrm{C}$ for $1 \mathrm{~min}$, using the 7900 Fast RT-PCR System (Applied Biosystems). 


\section{Statistical analysis}

All statistical analysis were carried out using the SPSS software (IBM, USA). Differences between two groups were determined using a paired $t$-test. The Wilcoxon-Mann-Whitney test was used to evaluate the relationships between the miR-125b expression level and clinicopathologic features of the subjects. The receiver operating characteristic curve (ROC) analysis was used to determine the diagnostic accuracy of serum miR-125b, with the area under the ROC (AUC) being used to determine the optimal sensitivity and specificity level. A P value of lower than 0.05 was considered to be statistically significant.

\section{RESULTS}

\section{General characteristics of subjects}

The demographic characteristics of all subjects are listed in Table 1. There were thirty-one and thirty-two patients whose tumor size was greater or lesser than $8 \mathrm{~cm}$, respectively. Distant metastases were observed only in 9 cases. Thirty-four ES patients presented poor response to chemotherapy.

Table 1. Correlation between miR-125b expression and different clinicopathologic features in ES patients.

\begin{tabular}{|c|c|c|c|c|}
\hline \multirow[t]{2}{*}{ Variables } & \multirow[t]{2}{*}{ No. of cases $(\mathrm{N}=63)$} & \multicolumn{2}{|c|}{ Relative expression of miR-125b* } & \multirow[t]{2}{*}{$P$ value } \\
\hline & & High & Low & \\
\hline \multicolumn{5}{|l|}{ Age (years) } \\
\hline$\geq 12$ vs $<12$ & $39 / 24$ & $14 / 9$ & $25 / 15$ & 0.898 \\
\hline \multicolumn{5}{|l|}{ Gender } \\
\hline Male vs female & $46 / 17$ & $18 / 5$ & $28 / 12$ & 0.477 \\
\hline \multicolumn{5}{|l|}{ Tumour size $(\mathrm{cm})$} \\
\hline$\geq 8$ vs $<8$ & $31 / 32$ & $13 / 10$ & $18 / 22$ & 0.378 \\
\hline \multicolumn{5}{|l|}{ Tumour site } \\
\hline Axial vs extremity & $21 / 42$ & 9/14 & $12 / 28$ & 0.459 \\
\hline \multicolumn{5}{|l|}{ Tumor metastasis } \\
\hline Yes vs no & 9/54 & $4 / 19$ & $5 / 35$ & 0.593 \\
\hline \multicolumn{5}{|l|}{ RTC } \\
\hline Good vs poor & $29 / 34$ & $17 / 6$ & $12 / 28$ & 0.001 \\
\hline
\end{tabular}

RTC = response to chemotherapy. ${ }^{*}$ The median expression level of circulating miR-125b was used as the cut-off value to categorize the patients into high- and low-expression groups.

\section{Serum expression of $\mathrm{miR}-125 \mathrm{~b}$}

Expression of serum miR-125b was distinctly decreased in ES patients when compared with healthy controls $(P<0.001$; Figure 1$)$. Subsequently, the correlation between the expression levels of miR-125b and the clinicopathological features of the ES cases was analyzed (Table 1). According to the expression levels of miR-125b using a cut-off value of 2.203, ES patients were divided into two groups (high and low expression groups). With respect to most of the clinicopathological features, no significant differences were found in the correlations of miR-125b expression levels with clinicopathological features, including age, gender, tumor size, tumor site, and tumor metastasis. However, ES cases who had a poor response to chemotherapy presented a significant down-regulation of $\mathrm{miR}-125 \mathrm{~b}(\mathrm{P}=0.001)$. 


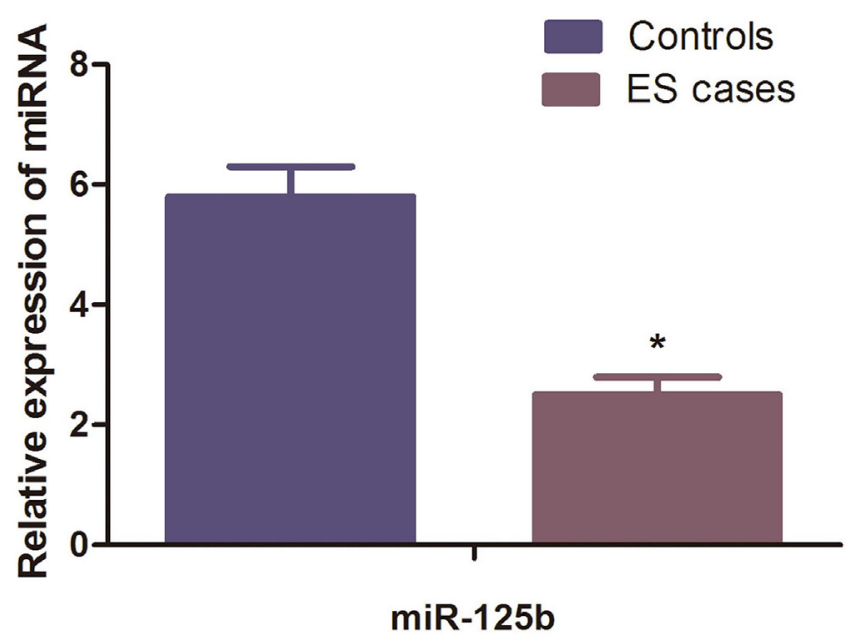

Figure 1. Relative expression level of miR-125b in healthy controls and ES patients. The expression levels were determined using a qRT-PCR assay, and the relative expression data were measured using the $2^{-\Delta \Delta C t}$ method. All of the assays were performed in triplicate. U6 was used as a reference miR. ${ }^{*} \mathrm{P}<0.001$.

\section{Diagnostic accuracy of serum miR-125b for ES}

The ROC curve was calculated to confirm the diagnostic accuracy of serum miR-125b for ES. The results showed that the serum miR-125b could serve as a valuable biomarker to differentiate ES patients from healthy controls with an AUC of $0.879(95 \% \mathrm{Cl}=0.817-0.924 ; \mathrm{P}$ $<0.001$; Figure 2). At a cut-off value of 2.203 for miR-125b, the sensitivity was $72.8 \%$ and the specificity was $87.2 \%$ for discriminating ES from the controls.

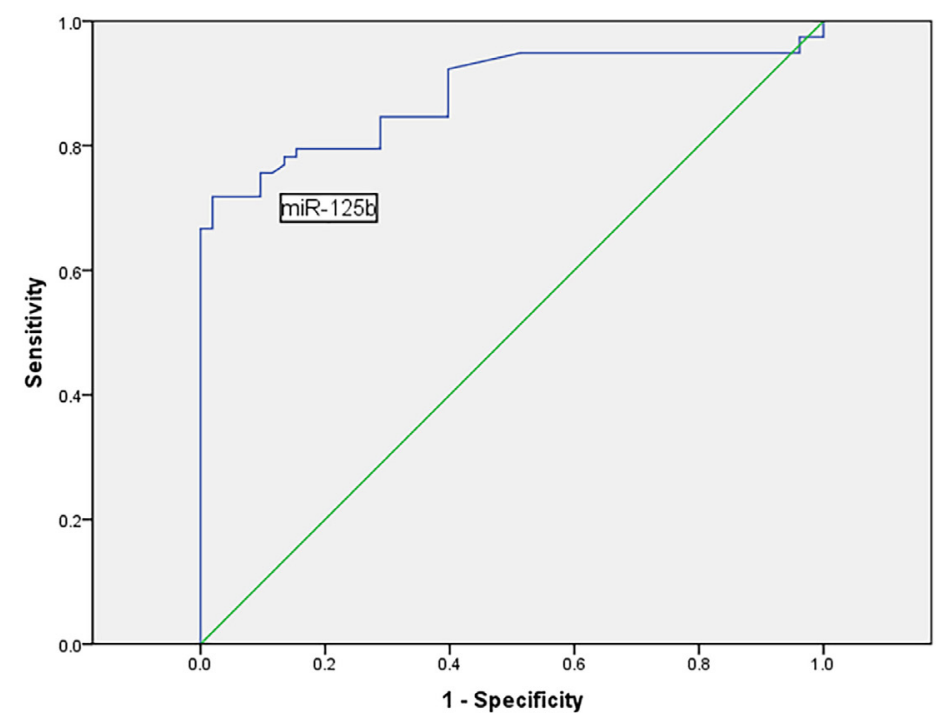

Figure 2. Receiver operating curve (ROC) analysis of miR-125b expression level. 


\section{DISCUSSION}

The search for non-invasive biomarkers for the diagnosis of ES is currently one of the most rapidly growing areas in ES research (Marino et al., 2014). Over the past decades, researchers and clinicians have undertaken long-range efforts in early screening and multimodal regimens, including local surgery, radiotherapy, and intensive systemic chemotherapy (Bacci et al., 2006; Balamuth and Womer, 2010). However, the presence of clinically evident metastasis at diagnosis and overall survival of ES patients remain quite dismal. Although many biological and molecular markers have been investigated (Riley et al., 2003; van Maldegem et al., 2012), their practical value in clinical work still requires validation and experimental configuration.

In numerous recent studies, in the blood serum, circulating miRs were detected, which are stable, reproducible, resistant to several harsh conditions, and have already been recommended as non-invasive novel biomarkers for the diagnosis of many malignant tumors (Miller et al., 2013). Recent reports have already given more prominence to the oncogenic aspects of miR$125 \mathrm{~b}$, claiming that serum miR-125b could be a novel biomarker for screening human tumors, including non-small-cell lung cancer and breast cancer (Wang et al., 2012; Yuxia et al., 2012). Our previous miR array analysis in ES tissue samples has demonstrated the diagnostic potential of miR-125b (Li et al., 2014). Therefore, it is possible that miR-125b could become a biomarker for the diagnosis of ES.

Here, serum miR-125b was studied in patients with ES at initial diagnosis and compared with healthy controls. Serum miR-125b level was significant decreased in ES patients when compared to healthy individuals. Furthermore, our findings also indicated that miR-125b alone might serve as a novel, noninvasive biomarker for ES with a high sensitivity of $72.8 \%$ and specificity of $87.2 \%$.

Many recent studies reported that the specific profiles of serum miRs might serve as novel, noninvasive biomarkers for ES. To date, previous studies (Sato et al., 2011; Mosakhani et al., 2012; Sasaki et al., 2013; Karnuth et al., 2014; Todorova, 2014) with microarray analysis indicated significant overexpression of five miRs (miR-106b, miR-93, miR-181b, miR-101, and miR-30b), while significant underexpression of six miRs (miR-145, miR-193a-3p, miR-100, miR-22, miR-21, and miR-574-3p) in ES tissues when compared to control samples. Ban et al. (2011) showed that, as a tumor suppressor, miR-145 replacement leads to the inhibition of anchorage-independent growth of ES cells. Nakatani et al. (2012) revealed that five miRs (miR-34a, miR-23a, miR-92a, miR-490-3p, and miR-130b) might serve as independent risk predictors for the progression and survival of ES. To date, despite the dysregulation of miRs in ES tissues in overwhelming reports, no serum miR has been investigated as a noninvasive biomarker for the diagnosis of ES. Our findings revealed that the identification of $\mathrm{miR}-125 \mathrm{~b}$ might contribute to this purpose.

Several limitations of our study should not be ignored. The sample size is relatively modest, which might cause selection bias for patients. Second, replicated investigations in other groups were not carried out. Then, other potential relevant miRs, which play important roles in ES as well, should also be investigated. Finally, the exact mechanisms of miR-125b should be identified in further studies.

In summary, we provided evidence that serum miR-125b levels were significantly decreased in ES patients, and serum ES level was significantly correlated with chemotherapy. Moreover, with the findings of its reasonable sensitivity and specificity for ES, miR-125b could become a useful biomarker for ES diagnosis. Nonetheless, further studies with larger sample size involving the exact mechanism should be carried out. 


\title{
Conflicts of interest
}

The authors declare no conflict of interest.

\section{ACKNOWLEDGMENTS}

\author{
Research supported by Shandong University.
}

\section{REFERENCES}

Bacci G, Longhi A, Ferrari S, Mercuri M, et al. (2006). Pattern of relapse in 290 patients with nonmetastatic Ewing's sarcoma family tumors treated at a single institution with adjuvant and neoadjuvant chemotherapy between 1972 and 1999 . Eur. J. Surg. Oncol. 32: 974-979.

Balamuth NJ and Womer RB (2010). Ewing's sarcoma. Lancet Oncol. 11: 184-192.

Ban J, Jug G, Mestdagh P, Schwentner R, et al. (2011). Hsa-mir-145 is the top EWS-FLI1-repressed microRNA involved in a positive feedback loop in Ewing's sarcoma. Oncogene 30: 2173-2180.

Barr RD and Wunder JS (2009). Bone and soft tissue sarcomas are often curable - but at what cost?: a call to arms (and legs). Cancer 115: 4046-4054.

Bartel DP (2004). MicroRNAs: genomics, biogenesis, mechanism, and function. Cell 116: 281-297.

Borinstein SC, Beeler N, Block JJ, Gorlick R, et al. (2013). A Decade in Banking Ewing Sarcoma: A Report from the Children's Oncology Group. Front. Oncol. 3: 57.

De Vito C, Riggi N, Suva ML, Janiszewska M, et al. (2011). Let-7a is a direct EWS-FLI-1 target implicated in Ewing's sarcoma development. PLoS One 6: e23592.

Dorfman HD and Czerniak B (1995). Bone cancers. Cancer 75 (Suppl 1): 203-210.

Erisir F, Güvenc MG, Dervisoglu S, Oz B, et al. (2008). Ewing/Primitive neuroectodermal tumour family sarcoma arising from a branchial cyst. J. Otolaryngol. Head Neck Surg. 37: E96-E99.

Esiashvili N, Goodman M and Marcus RB Jr. (2008). Changes in incidence and survival of Ewing sarcoma patients over the past 3 decades: Surveillance Epidemiology and End Results data. J. Pediatr. Hematol. Oncol. 30: 425-430.

Fabbri M (2010). miRNAs as molecular biomarkers of cancer. Expert Rev. Mol. Diagn. 10: 435-444.

Ferracin M, Veronese A and Negrini M (2010). Micromarkers: miRNAs in cancer diagnosis and prognosis. Expert Rev. Mol. Diagn. 10: 297-308.

Friedman RC, Farh KK, Burge CB and Bartel DP (2009). Most mammalian mRNAs are conserved targets of microRNAs. Genome Res. 19: 92-105.

Ghildiyal M and Zamore PD (2009). Small silencing RNAs: an expanding universe. Nat. Rev. Genet. 10: 94-108.

lida K, Fukushi J, Matsumoto Y, Oda Y, et al. (2013). miR-125b develops chemoresistance in Ewing sarcoma/primitive neuroectodermal tumor. Cancer Cell Int. 13: 21.

Iwamoto Y (2007). Diagnosis and treatment of Ewing's sarcoma. Jpn. J. Clin. Oncol. 37: 79-89.

Karnuth B, Dedy N, Spieker T, Lawlor ER, et al. (2014). Differentially expressed miRNAs in Ewing sarcoma compared to mesenchymal stem cells: low miR-31 expression with effects on proliferation and invasion. PLoS One 9: e93067.

Kozomara A and Griffiths-Jones S (2011). miRBase: integrating microRNA annotation and deep-sequencing data. Nucleic Acids Res. 39: D152-D157.

Li J, You T and Jing J (2014). MiR-125b inhibits cell biological progression of Ewing's sarcoma by suppressing the PI3K/Akt signalling pathway. Cell Prolif. 47: 152-160.

Marino MT, Grilli A, Baricordi C, Manara MC, et al. (2014). Prognostic significance of miR-34a in Ewing sarcoma is associated with cyclin D1 and ki-67 expression. Ann. Oncol. 25: 2080-2086.

McKinsey EL, Parrish JK, Irwin AE, Niemeyer BF, et al. (2011). A novel oncogenic mechanism in Ewing sarcoma involving IGF pathway targeting by EWS/Fli1-regulated microRNAs. Oncogene 30: 4910-4920.

Miller IV, Raposo G, Welsch U, Prazeres da Costa O, et al. (2013). First identification of Ewing's sarcoma-derived extracellular vesicles and exploration of their biological and potential diagnostic implications. Biol. Cell. 105: 289-303.

Mosakhani N, Guled M, Leen G, Calabuig-Fariñas S, et al. (2012). An integrated analysis of miRNA and gene copy numbers in xenografts of Ewing's sarcoma. J. Exp. Clin. Cancer Res. 31: 24.

Nakatani F, Ferracin M, Manara MC, Ventura S, et al. (2012). miR-34a predicts survival of Ewing's sarcoma patients and directly influences cell chemo-sensitivity and malignancy. J. Pathol. 226: 796-805. 
Riley RD, Burchill SA, Abrams KR, Heney D, et al. (2003). A systematic review of molecular and biological markers in tumours of the Ewing's sarcoma family. Eur. J. Cancer 39: 19-30.

Sasaki T, Onishi T, Yabana T and Hoshina A (2013). Ewing's sarcoma/primitive neuroectodermal tumor arising from the adrenal gland: a case report and literature review. Tumori 99: e104-e106.

Sato F, Tsuchiya S, Meltzer SJ and Shimizu K (2011). MicroRNAs and epigenetics. FEBS J. 278: 1598-1609.

Schleiermacher G, Peter M, Oberlin O, Philip T, et al. (2003). Increased risk of systemic relapses associated with bone marrow micrometastasis and circulating tumor cells in localized ewing tumor. J. Clin. Oncol. 21: 85-91.

Stahl M, Ranft A, Paulussen M, Bölling T, et al. (2011). Risk of recurrence and survival after relapse in patients with Ewing sarcoma. Pediatr. Blood Cancer 57: 549-553.

Todorova R (2014). Ewing's sarcoma cancer stem cell targeted therapy. Curr. Stem Cell Res. Ther. 9: 46-62.

van Maldegem AM, Hogendoorn PC and Hassan AB (2012). The clinical use of biomarkers as prognostic factors in Ewing sarcoma. Clin. Sarcoma Res. 2: 7.

Wang H, Tan G, Dong L, Cheng L, et al. (2012). Circulating MiR-125b as a marker predicting chemoresistance in breast cancer. PLoS One 7: e34210.

Yu SL, Chen HY, Yang PC and Chen JJ (2007). Unique MicroRNA signature and clinical outcome of cancers. DNA Cell Biol. 26: 283-292.

Yuxia M, Zhennan T and Wei Z (2012). Circulating miR-125b is a novel biomarker for screening non-small-cell lung cancer and predicts poor prognosis. J. Cancer Res. Clin. Oncol. 138: 2045-2050. 